\title{
Rigorous electromagnetic simulation of mask magnification effects on the diffracted light for EUV binary mask
}

\author{
Chun-Hung Lin ${ }^{\mathrm{a}, *}$, Hsuen-Li Chen ${ }^{\mathrm{b}}$, Fu-Hsiang Ko ${ }^{\mathrm{c}}$ \\ ${ }^{a}$ National Nano Device Laboratories, Hsinchu 300, Taiwan \\ ${ }^{\mathrm{b}}$ Department of Materials Science and Engineering, National Taiwan University, Taipei 106, Taiwan \\ ${ }^{\mathrm{c}}$ Institute of Nanotechnology, National Chiao Tung University, Hsinchu 300, Taiwan
}

Available online 25 January 2007

\begin{abstract}
Extreme ultraviolet (EUV) lithography is expected to be the main candidate in the semiconductor manufacturing starting at $32 \mathrm{~nm}$. As the CD is getting smaller, the aspect ratio of the patterns on the EUV mask is becoming larger. The shadowing effect will become much more significant when keeping the same $4 \times$ mask magnification. In this work, mask magnification effects on the diffracted light were explored with rigorous coupled-wave analysis (RCWA) for the sub-32 nm node. The simulated binary mask consists of 70 -nm TaBN absorber and 2.5-nm Ru capping layer. The dependences of the diffraction efficiencies on mask pitches were calculated. The impacts of the absorber shadowing were observed from the near field distribution on the EUV mask. The aerial images formed by the diffracted light from the $4 \times$ and $8 \times$ masks were further evaluated.
\end{abstract}

(C) 2007 Elsevier B.V. All rights reserved.

Keywords: Extreme ultraviolet lithography; Mask magnification; Rigorous coupled-wave analysis

\section{Introduction}

Extreme ultraviolet (EUV) lithography is expected to be the main candidate in the semiconductor manufacturing starting at $32 \mathrm{~nm}$ [1]. Due to the reflective mask and the reflective optics in EUV, the illuminating incident light should be oblique incidence. On the other hand, as the $\mathrm{CD}$ is getting smaller, the aspect ratio of the patterns on the EUV mask is becoming larger. The shadowing effect [2] will become much more significant when keeping the same $4 \times$ mask magnification as proposed in ITRS 2005 edition [1]. Moreover, the defect control at the $4 \times$ mask should be a severe problem. The mask cost would increase significantly because of the stringent mask critical dimension (CD) control and the defect problem at the mask blank. The change to higher mask magnification factor has been proposed to relax the above mentioned severe situations

\footnotetext{
* Corresponding author.

E-mail address: chlin@mail.ndl.org.tw (C.-H. Lin).
}

[3]. The mask cost was estimated be reduced to at least $50 \%$ at 32 -nm node. An $8 \times$ scanner with the double long mask could give almost the same relative throughput to $4 \times$ because the stage scanning speed is not the limiting factor in EUV lithography. In this work, mask magnification effects of the EUV binary mask on the diffracted light were evaluated for the sub-32 nm node. A rigorous coupledwave analysis (RCWA) [4,5] was developed and employed to analyze the optical diffraction from the $3 \mathrm{D}$ topographic periodic features.

\section{Analysis}

\subsection{Rigorous coupled-wave analysis}

The advantage of using the RCWA is that the reflected field from the mask has been expressed in terms of the spatial Fourier expansion, which can be directly employed in the following aerial image analysis. Fig. 1 illustrates the geometry of the RCWA configuration for the mask. The 


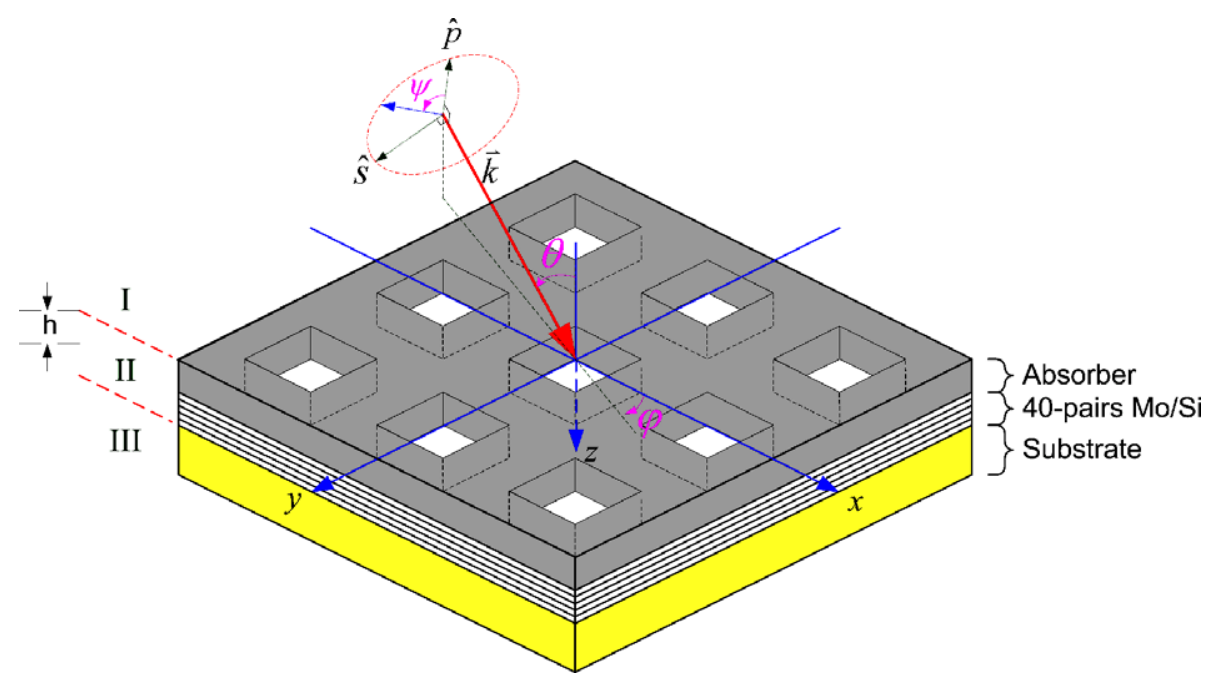

Fig. 1. The geometry of the RCWA configuration for the EUV mask.

whole structure can be divided into three regions: the incident region (Region I), the grating region (Region II) and the exit region (Region III). The incident normalized electric field can be expressed in the form

$\mathbf{E}_{\text {inc }}=\hat{u} \exp \left[-\mathrm{j}\left(k_{\mathrm{inc}, x} x+k_{\text {inc }, y} y+k_{\mathrm{inc}, z} z\right)\right]$

where $\hat{\mathrm{u}}$ is the unit polarization vector; $k_{\text {inc, } x}=k_{0} n_{\mathrm{I}} \sin \theta$ $\cos \varphi ; k_{\text {inc }, y}=k_{0} n_{\mathrm{I}} \sin \theta \sin \varphi ; k_{\text {inc }, z}=k_{0} n_{\mathrm{I}} \cos \theta ; k_{0}=2 \pi / \lambda$; and $n_{\mathrm{I}}$ is the refractive index of the incident region. The total electric fields in the external regions (I, III) can be expressed as

$$
\begin{aligned}
& \mathbf{E}_{\mathrm{I}}=\mathbf{E}_{\mathrm{inc}}+\sum_{m, n} \mathbf{R}_{m n} \exp \left[-\mathrm{j}\left(k_{x m} x+k_{y n} y-k_{\mathrm{I} z, m n} z\right)\right] \\
& \mathbf{E}_{\mathrm{III}}=\sum_{m, n} \mathbf{T}_{m n} \exp \left\{-\mathrm{j}\left[k_{x m} x+k_{y n} y+k_{\mathrm{III} z, m n}(z-h)\right]\right\}
\end{aligned}
$$

where $\mathbf{R}_{m n}$ and $\mathbf{T}_{m n}$ are the complex amplitudes of $(m, n)$ th reflected and transmitted orders, respectively. The wave vector components $k_{x m}$ and $k_{y n}$ can be determined from the Floquet conditions and are given by

$$
\begin{aligned}
& k_{x m}=k_{\text {inc }, x}+m\left(\frac{2 \pi}{\mathfrak{R} \Lambda_{x}}\right) \\
& k_{y n}=k_{\text {inc }, y}+n\left(\frac{2 \pi}{\mathfrak{R} \Lambda_{y}}\right) \\
& k_{x m}^{2}+k_{y n}^{2}+k_{l z, m n}^{2}=\left(k_{0} n_{l}\right)^{2}, \quad l=\mathrm{I}, \text { III }
\end{aligned}
$$

where $\mathfrak{R}$ is the reduction factor of the projection system. For example, $\mathfrak{R}$ is equal to 4 for the $4 \times$ reduction; $\Lambda_{x}$ and $\Lambda_{y}$ are the periods of the grating along $x$ and $y$ direction in the wafer scale. $n_{\mathrm{I}}$ and $n_{\mathrm{III}}$ are the refractive indices of the incident and exit regions.

The magnetic fields in the external regions can be obtained from Maxwell's equation

$\mathbf{H}=-\frac{1}{\mathrm{j} \omega \mu_{0}} \nabla \times \mathbf{E}$ where $\mu_{0}$ is the permeability of free space and $\omega$ is the angular optical frequency.

In the grating region, the periodic permittivity can be expressed in the Fourier expansion

$\varepsilon_{\text {III }}(x, y)=\sum_{g, h} \varepsilon_{g h} \exp \left[\mathrm{j} k_{0}\left(\frac{\lambda}{\mathfrak{R} \Lambda_{x}} g x+\frac{\lambda}{\mathfrak{R} \Lambda_{y}} h y\right)\right]$

The electric and magnetic fields in the grating region can be expressed with a Fourier expansion in terms of the spaceharmonic fields

$$
\begin{aligned}
& \mathbf{E}_{\mathrm{II}}=\sum_{m, n} \mathbf{S}_{m n}(z) \exp \left[-\mathrm{j}\left(k_{x m} x+k_{y n} y\right)\right] \\
& \mathbf{H}_{\mathrm{II}}=-\mathrm{j} \sqrt{\frac{\varepsilon_{0}}{\mu_{0}}} \sum_{m, n} \mathbf{U}_{m n}(z) \exp \left[-\mathrm{j}\left(k_{x m} x+k_{y n} y\right)\right]
\end{aligned}
$$

where $\varepsilon_{0}$ and $\mu_{0}$ are the permittivity and permeability of free space, respectively. $\mathbf{S}_{m n}$ and $\mathbf{U}_{m n}$ are the complex amplitudes of the $(m, n)$ th space-harmonic fields. Finally, by applying the Maxwell's equation in the grating region, matching the boundary conditions at the interfaces between different spatial regions, and solving the linear equation system with the $S$-matrix algorithm [6], the unknown amplitudes $\mathbf{R}_{m n}$ and $\mathbf{T}_{n m}$ of the diffracted waves can be determined.

The near field distribution of the periodic mask can be treated as the complex amplitude distribution of the object in the imaging system and is re-written directly from the Eq. (2)

$\mathbf{E}_{\mathrm{O}}(x, y)=\sum_{m, n} \mathbf{R}_{m n} \exp \left[-\mathrm{j}\left(k_{x m} x+k_{y n} y\right)\right]$

By considering the reduction factor $\mathfrak{R}$ and image inversion, the ideal image can be further expressed as

$$
\begin{aligned}
\mathbf{E}_{\mathrm{G}}(\tilde{x}, \tilde{y}) & =\mathfrak{R} \mathbf{E}_{\mathrm{O}}(-\mathfrak{R} \tilde{x},-\mathfrak{R} \tilde{y}) \\
& =\mathfrak{R} \sum_{m, n} \mathbf{R}_{m n} \exp \left[\mathrm{j} \mathfrak{R}\left(k_{x m} \tilde{x}+k_{y n} \tilde{y}\right)\right]
\end{aligned}
$$


where $(\tilde{x}, \tilde{y})=-(x, y) / \Re$ are the reduced coordinates in the object plane. Finally, considering the finite bandpass of the optical system

$\mathbf{E}_{\mathrm{I}}(x \prime, y \prime)=F^{-1}\left\{H\left(f_{x}, f_{y}\right) \mathbf{E}_{\mathrm{G}}\left(f_{x}, f_{y}\right)\right\}$

where

$\mathbf{E}_{\mathrm{G}}$ is the spatial spectrum of the ideal image obtained with the Fourier transform operation.

$$
\begin{aligned}
\mathbf{E}_{\mathrm{G}}\left(f_{x}, f_{y}\right) & =F\left\{\mathbf{E}_{\mathrm{G}}(\tilde{x}, \tilde{y})\right\} \\
& =\mathfrak{R} \sum_{m, n} \mathbf{R}_{m n} \delta\left(f_{x}-\mathfrak{R} k_{x m}\right) \delta\left(f_{y}-\mathfrak{R} k_{y n_{n}}\right)
\end{aligned}
$$

The spatial frequencies of the $(m, n)$ th diffracted orders from the exit pupil of the optical system are $\left(\mathfrak{R} k_{x m}, \mathfrak{R} k_{y n}\right)$ and the $\mathbf{R}_{m n}$ can be treated as the corresponding complex amplitudes.

$H$ is the scalar pupil function and is band-limited by the $\mathrm{NA}$ of the lens.

$H\left(f_{x}, f_{y}\right)=0$ for $\sqrt{f_{x}^{2}+f_{y}^{2}}>k_{0} N A$

\subsection{Simulated mask structure}

Fig. 2 shows the EUV binary mask structure in our simulation. The mask substrate was assumed to consist of 40layer pairs of Mo and Si with layer thickness of 2.8 and $4.15 \mathrm{~nm}$, respectively. A Ru capping layer of $2.5 \mathrm{~nm}$ and a TaBN absorber of $70 \mathrm{~nm}$ were used in the simulation $[7,8]$. Assuming the use of a damage-free mask repair technology [9], no buffer layer is considered to minimize the shadowing effects. The optical constants of all materials were obtained from the database [10]. The illumination angle was set to be $6^{\circ}$ in all the following simulations if not especially mentioned. Since the light passing through the mask is a relatively small angle to the optical axis in EUV lithography, the polarization effect is not significant. Therefore, only TE-polarized light was considered in the simulation.

\section{Results}

\subsection{Diffraction efficiency dependencies on pitch}

Fig. 3 shows the diffraction efficiencies of the line/space patterns with 1:1 line to space ratio for the $\mathrm{CD}$ from

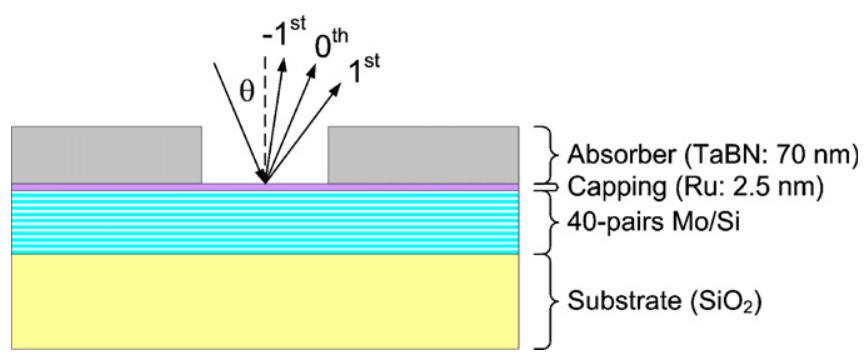

Fig. 2. The simulated binary mask structure. The mask consists of $70-\mathrm{nm}$ $\mathrm{TaBN}$ absorber and 2.5-nm Ru capping layer.

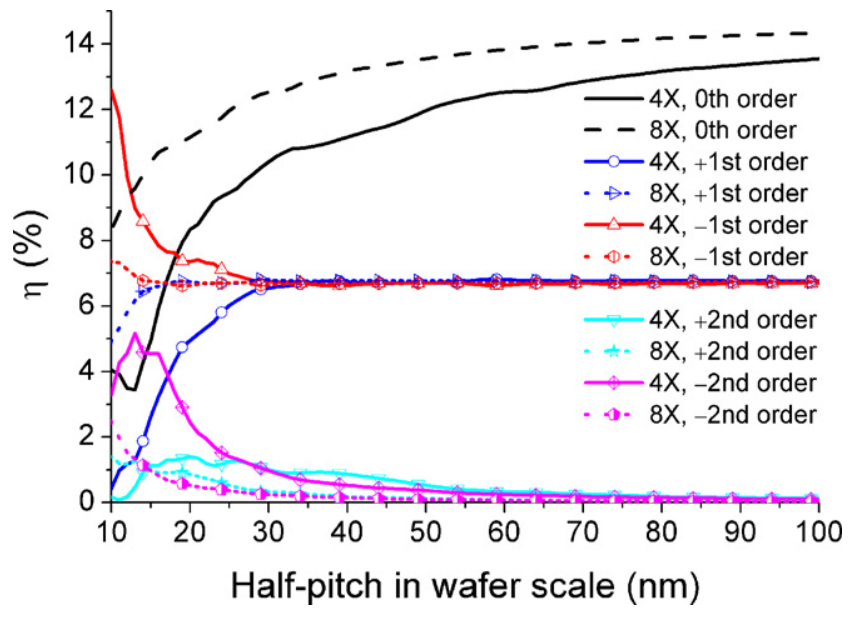

Fig. 3. Diffraction efficiencies of the line/space patterns with 1:1 line to space ratio for the CD from $10 \mathrm{~nm}$ to $100 \mathrm{~nm}$.

$10 \mathrm{~nm}$ to $100 \mathrm{~nm}$. The mask magnification factors with $4 \times$ and $8 \times$ were compared and the dimensions were normalized to the wafer scale. For the $4 \times$ mask, the diffraction efficiency of the 0 th reflected light is $13.55 \%$ in the $100-\mathrm{nm}$ linewidth, $10.70 \%$ in the $32-\mathrm{nm}$ linewidth, and decreases to $5.94 \%$ in the 16-nm linewidth. The diffraction efficiencies of the \pm 1 st reflected lights keep the almost the same value at around $6.70 \%$ for the $\mathrm{CD}$ larger than $32 \mathrm{~nm}$. In a CD of $16 \mathrm{~nm}$, the -1 st diffraction efficiency increases to $7.84 \%$ and the +1 st diffraction efficiency decreases to $3.24 \%$. The diffraction efficiencies of the \pm 2 nd reflected lights are smaller than $1 \%$ for the CD larger than $32 \mathrm{~nm}$. In a CD of $16 \mathrm{~nm}$, the -2 nd diffraction efficiency increases to $4.54 \%$ and the +2 nd diffraction efficiency increases to $1.13 \%$. Although there are stronger diffraction efficiencies in \pm 2 nd reflected lights in small CDs, the \pm 2 nd reflected lights cannot participate in the image formulation because of their larger diffraction angles. From Eq. (3), the difference of the spatial frequency between each diffracted light are $\lambda /$ pitch. Fig. 4 shows the dependency of $\lambda /$ pitch on the half-pitch. The value of $\lambda /$ pitch is 0.31 in the 22 -nm linewidth and 0.42 in the 16-nm linewidth. Therefore, only two diffracted beams

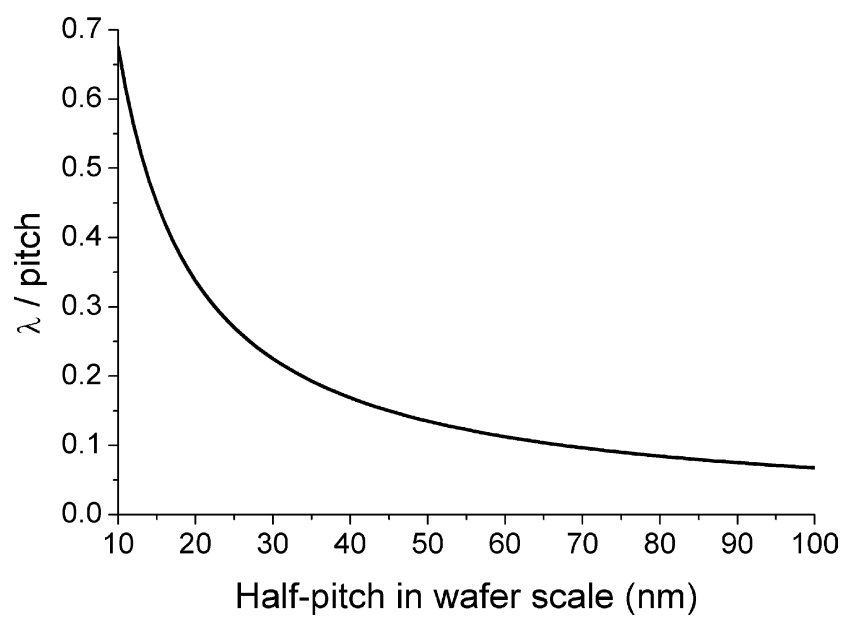

Fig. 4. Dependency of $\lambda /$ pitch on the half-pitch. 
can pass the exit pupil of the EUV optical system with a NA of 0.3 . The effects of the 2 nd beams can be ignored in the sub-32 nm node even in an off-axis illumination. For the 16-nm linewidth in the $8 \times$ mask, the diffraction efficiencies can keep at $10.70 \%$ for the 0 th reflected light and $6.70 \%$ for the \pm 1 st reflected lights.

\subsection{Shadowing effect}

Because of the oblique illumination on EUV masks, the geometrical shadowing effect by the patterned absorber is

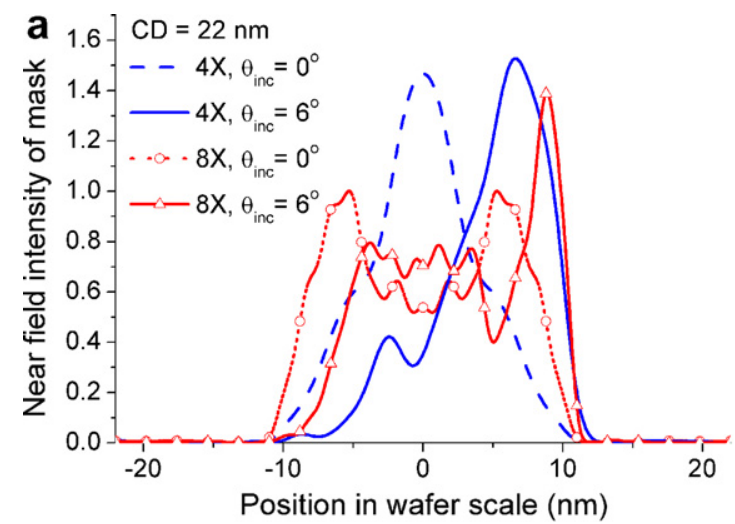

an inevitable problem. To see the intensity distributions after the exposing light reflected from the 3D topographic features, the near field distributions of masks were calculated as shown in Fig. 5. The patterns are the line/space patterns with 1:1 line to space ratio for a CD of $22 \mathrm{~nm}$ and $16 \mathrm{~nm}$, respectively. The cases of the illumination with a normal incidence were also simulated for comparison. For the $4 \times$ mask, the ratio of the absorber thickness over the linewidth is approaching to 1 in the sub- $32 \mathrm{~nm}$ node. The ratio is 0.80 in the $22-\mathrm{nm} \mathrm{CD}$ and 1.09 in the $16-\mathrm{nm}$ CD. In such a high ratio, the reflected light tends to con-

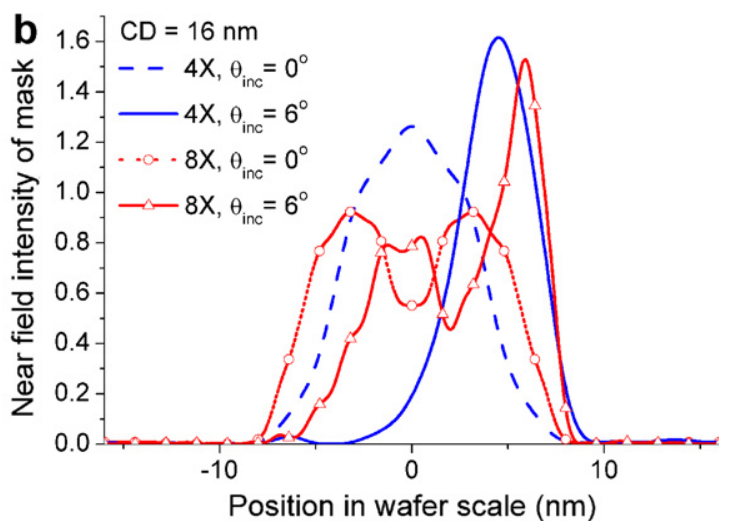

Fig. 5. Near field intensity of (a) $22-\mathrm{nm} \mathrm{CD}$ and (b) 16-nm CD in wafer scale from the $4 \times$ and $8 \times$ masks. The patterns are the line/space patterns with $1: 1$ line to space ratio. The illuminating angles of $0^{\circ}$ and $6^{\circ}$ were compared.
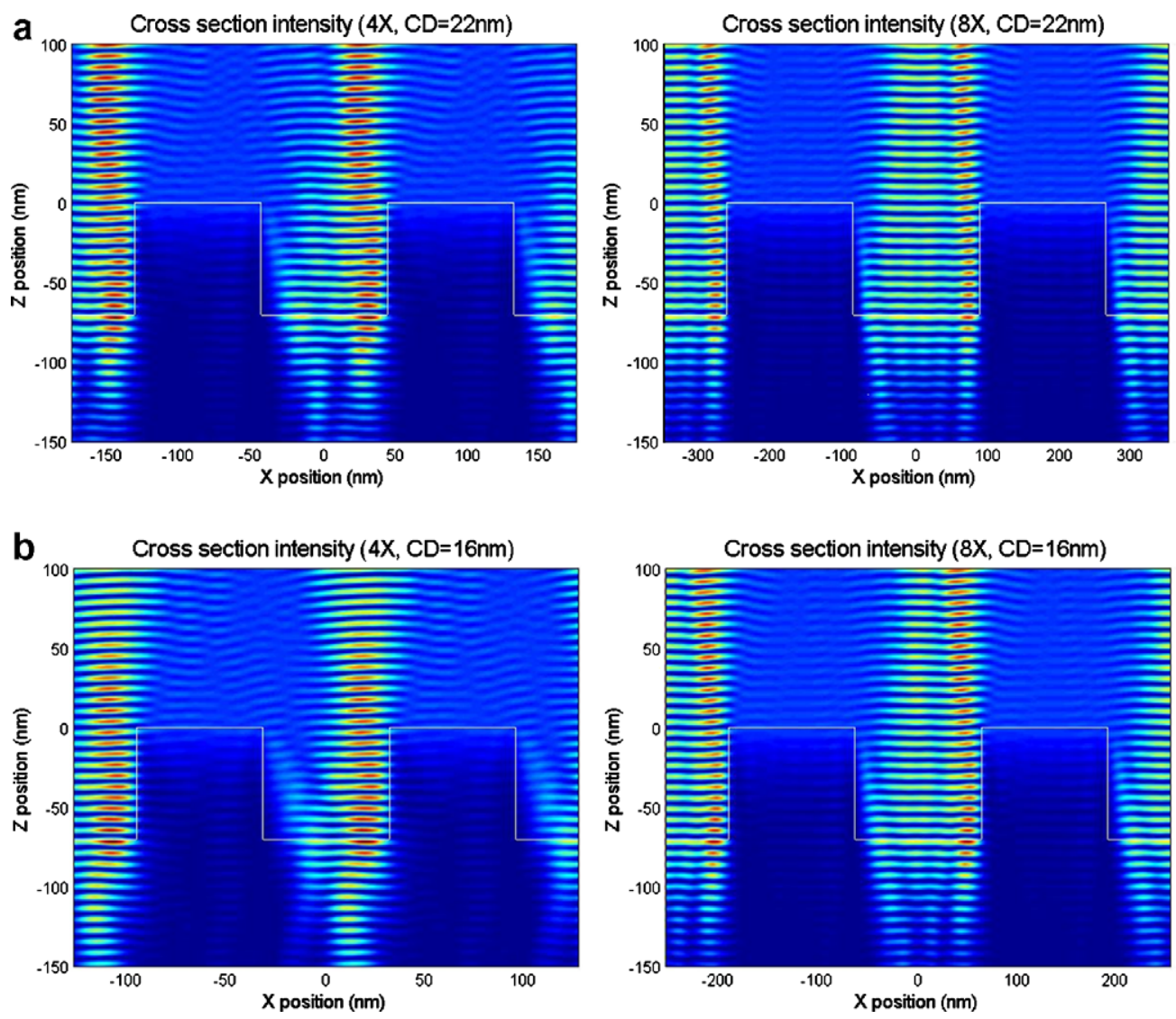

Fig. 6. Cross section view of the near field distribution of (a) $22-\mathrm{nm} \mathrm{CD}$ and (b) $16-\mathrm{nm} \mathrm{CD}$ in wafer scale from the $4 \times$ and $8 \times$ masks. The patterns are the line/space patterns with 1:1 line to space ratio. 

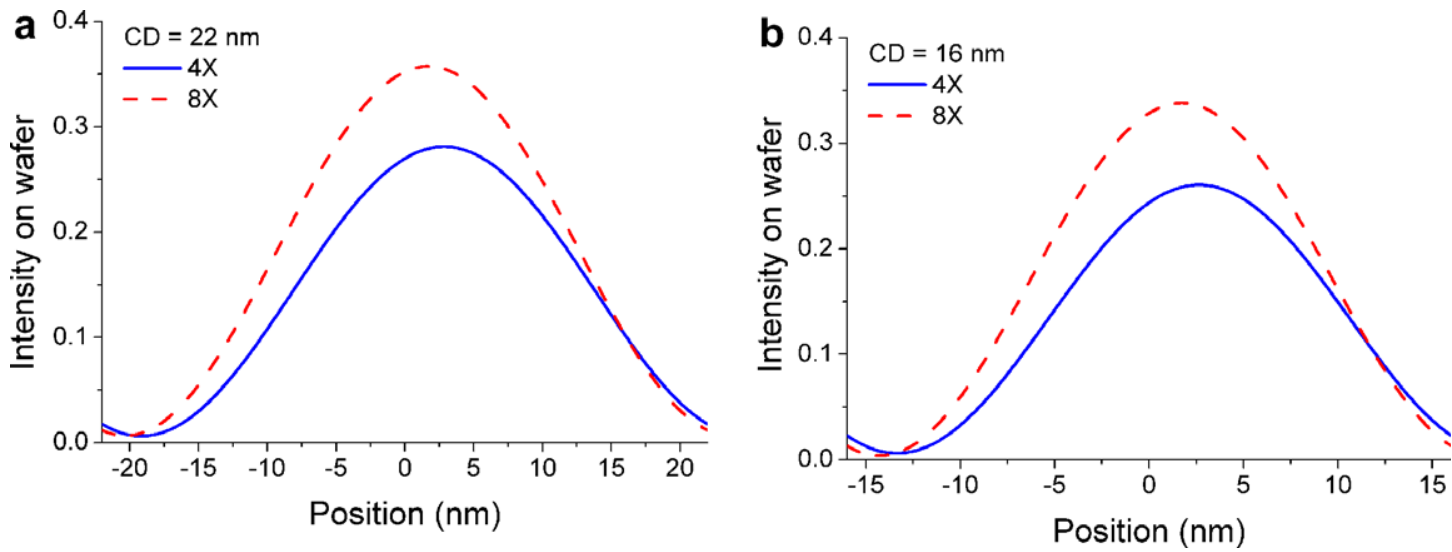

Fig. 7. Aerial images of (a) 22-nm CD and (b) $16-\mathrm{nm}$ CD from the $4 \times$ and $8 \times$ masks. The patterns are the line/space patterns with 1:1 line to space ratio. The NA of the EUV system was assumed to be 0.3 and the illumination is in the dipole configuration with $\sigma_{\text {center }}=0.7$ and $\sigma_{\text {radius }}=0$.

centrate on the center of the reflected region in a normal incident illumination. The center peak intensity is shifted toward to one absorber edge when the illuminating angle is $6^{\circ}$. The shifting phenomenon is stronger for a smaller CD. For the $8 \times$ mask, the reflected light tends to spread over the reflected region because of the smaller ratio of the absorber thickness over the linewidth. There are two stronger peaks in the edge when the illumination is in a normal incidence. When the illuminating angle is $6^{\circ}$, the reflected light is also shifted toward to one absorber edge, but the shift is not as strong as the case of the $4 \times$ mask. The peak in the shifting direction is enhanced and another peak in the opposite side is suppressed. Fig. 6 shows the cross section view of the near field distribution on the EUV mask. The asymmetric phenomenon of the diffracted light in the $4 \times$ mask is much stronger as compared with that in the $8 \times$ mask.

\subsection{Imaging performance}

The aerial images formed by the diffracted light from the $4 \times$ and $8 \times$ masks were also evaluated as shown in Fig. 7. The patterns are the line/space patterns with 1:1 line to space ratio for a CD of $22 \mathrm{~nm}$ and $16 \mathrm{~nm}$, respectively. The NA of the EUV system was assumed to be 0.3 and the illumination is in the dipole configuration with $\sigma_{\text {cen- }}$ ter $=0.7$ and $\sigma_{\text {radius }}=0$. The diffracted beams from the mask illuminated by each point source were rigorously calculated with RCWA. The images are normalized to the open frame intensity, which is the intensity of a clear mask without any feature. In a CD of $22 \mathrm{~nm}$, the intensity peak of the $4 \times$ mask is shifted $2.85 \mathrm{~nm}$, which is $12.95 \%$ of the linewidth. The intensity peak of the $8 \times$ mask is shifted $1.7 \mathrm{~nm}$, which is $7.73 \%$ of the linewidth. The peak intensity of the $8 \times$ mask is $27.2 \%$ stronger than that of the $4 \times$ mask. In a $C D$ of $16 \mathrm{~nm}$, the intensity peak of the $4 \times$ mask is shifted $2.65 \mathrm{~nm}$, which is $16.56 \%$ of the linewidth. The intensity peak of the $8 \times$ mask is shifted $1.7 \mathrm{~nm}$, which is $10.65 \%$ of the linewidth. The peak intensity of the $8 \times$ mask is $30.8 \%$ stronger than that of the $4 \times$ mask.

\section{Conclusion}

In this study, mask magnification effects on the diffracted light were evaluated rigorously with RCWA for the sub-32 nm node. The simulated binary mask consists of 70-nm TaBN absorber and 2.5-nm Ru capping layer. For the half-pitch smaller than $32 \mathrm{~nm}$ in the $4 \times$ mask, the diffraction efficiencies of 1:1 line/space patterns are deviated apparently from those at larger pitches. This phenomenon can be lowered to $16 \mathrm{~nm}$ in the $8 \times$ mask. The asymmetric phenomenon on the diffracted light in the $4 \times$ mask is much stronger because of the geometrical shadowing effect. With the introduction of the $8 \times$ mask, the lineedge shift at wafer can be reduced and image intensity is stronger when comparing with those of the $4 \times$ mask.

\section{Acknowledgments}

This work was supported by National Science Council, Taiwan, ROC (Grant No. 05095Z9000). The authors are grateful to the National Center for High-performance Computing for the support of the computing facilities.

\section{References}

[1] http://www.itrs.net/Links/2005ITRS/Home2005.htm.

[2] M. Besacier, P. Schiavone, Proc. SPIE 5446 (2004) 849-859.

[3] K. Takehisa, Proc. SPIE 5853 (2006) 874-883.

[4] M.G. Moharam, T.K. Gaylord, J. Opt. Soc. Am. 73 (1983) 11051112.

[5] C.H. Lin, H.L. Chen, W.C. Chao, C.I. Hsieh, W.H. Chang, Microelectron. Eng. 83 (2006) 1798-1804.

[6] L. Li, J. Opt. Soc. Am. A 13 (1996) 1024-1035.

[7] A.R. Stivers, P.Y. Yan, G. Zhang, T. Liang, E.Y. Shu, E. Tejnil, B. Lieberman, R. Nagpal, K. Hsia, M. Penn, F.C. Lo, Proc. SPIE 5567 (2004) 13-22.

[8] J.M. Roberts, T. Bacuita, R.L. Bristol, H.B. Cao, M. Chandhok, S.H. Lee, E.M. Panning, M. Shell, G. Zhang, B.J. Rice, Proc. SPIE 5751 (2005) 64-77.

[9] T. Liang, E. Frendberg, B. Lieberman, A.N. Stivers, J. Vac. Sci. Technol. B 23 (2005) 3101-3105.

[10] http://www-cxro.lbl.gov./optical_constants/getdb2.html. 